\title{
Flow onset of fresh mortars in rheometers: contribution ofpaste deflocculation and sand particle migration
}

\author{
Ye Qian and Shiho Kawashima*
}

Columbia University, Department of Civil Engineering and Engineering Mechanics, 500 West 120th street, New York, NY, 10027, USA

*Corresponding author at: Columbia University, Department of Civil Engineering and Engineering Mechanics, 500 West 120th street, 616 MUDD, New York, NY, 10027, USA.

Tel.: +1 2128542701

Email address: s-kawashima@columbia.edu

\begin{abstract}
Shear-inducedparticle migration is widely recognized to be a challenge in characterizing the rheological properties of fresh cement-based mortars. In this study, we aim to quantify shearinduced particlemigration by characterizing the stress decay process during constant shear flow with the aid of a modified thixotropy/migration model. It is found that a conventionally used single exponential model is not sufficient to fit the stress decay and describe the destructuration and sand migrationof mortar under shear. Instead, a two exponential model is needed to capture the interaction of sand particles and the suspending cement paste phase.Model parameters are used to quantify the effect of sand volume fraction, clay addition, and applied shear rate on thekinetics and intensity of colloidal deflocculation and sand migration. Results provide evidence that the colloidal and granular contributions to the overall stress decay of mortars can be represented by each of the two exponentials.
\end{abstract}

Keywords: Rheology (A);Modelling (E); Mortar (E);Aggregate (D); Sand migration.

\section{Introduction and Background}

Most studies on the rheology of cement-based materials thus far have focused on the paste phase and the concrete phase[1]. Paste is investigated because it is the phase that embodies the colloidal and 
hydration effects. However, the rheology of concrete is heavily influenced by the characteristics of the granular phase. Many experimental rheological studies investigate concrete systems directly to capture the critical aspects of particle packing and grain-to-grain contact. Sophisticated measurements are challenging, though, due to the limited sensitivity of large-scale viscometers and increased likeliness of inhomogeneity within the suspension, although some modified geometries have been proposed [1-3]. Mortar is an intermediate scale that exhibits both colloidal and granular behaviour. They can be prepared in relatively small batches, and tested on rotational rheometers with precise shear and measurement control, allowing for more complex flow situations. Serving as the suspending phase for the coarse aggregates, the rheological properties of the mortar are important in regards to the stability of fresh concrete systems[4]. Particularly for self-consolidating concrete (SCC),the mortar phase makes up a greater part of its composition compared to conventional concrete. Thus testing the rheological properties of mortar is an integral part of SCC design [5].

However, a widely recognized challenge in characterizing fresh cement mortar through shear rheological methods is shear-induced particle migration [6-8]. This has invoked studies that explore the influence of various parameters, e.g. setup geometries and solid inclusions, on sedimentation and migration under rotational shear [9]. However, more investigation is needed.In particular, it would be useful to develop a method to quantify sand particle migration in fresh mortarsto help guide the design of protocols for dynamic rheological characterization. Further, it can help determine the range of shear rates within which migration can be minimized or held constant. It can also be used to evaluate the effect of various mix parameters, e.g. mix proportioning and use of mineral/chemical admixtures, on dynamic segregation. As part of a wider investigation on the thixotropy of fresh mortar systems, in the present paper we discuss the potential of a thixotropy/migration model to quantify shear-induced particle migration in fresh mortars. 
The idealized shear stress response of a fresh cement-based suspension under a constant intermediate shear rate can be described as follows: an initial increaseto a peak value, considered to be the static yield stress as measured bythe stress growth protocol, followed by adecayuntil steady-state is reached. The shear stress decay from the peak value to the equilibrium value captures the process of structural breakdown and is related to the thixotropy of the material.Thixotropy is defined as a decrease in viscosity under shear, followed by an increase upon removal of shear. From a microstructural point of view, it can be described as paste deflocculation and breakage of CSH bridges under flow, and reflocculation and formation of $\mathrm{CSH}$ bridges over time at rest[10]. Meanwhile, sand migration induces structural heterogeneity. In investigating stress decay, we focus on paste deflocculation and sand migration.

The stress decay curve of cement pasteis found to be fitted well by an exponential curve. Empirically, Tattersall [11], Papo [12]and Lapasin et al. [13] measured the difference between the maximum shear stress, $\tau_{\max }$, needed to initiate flow and the steady-state equilibrium value, $\tau_{\mathrm{e}}$, at constant shear rate, then proposed simple thixotropy models thatpredict an exponential decrease of the shear stress. A logarithmic evolution of the shear stress was obtained, as follows:

$$
\tau=\tau_{\mathrm{e}}+\left(\tau_{\max }-\tau_{\mathrm{e}}\right) \exp (-\mathrm{Bt})
$$

Eq. 1

where $\mathrm{B}$ is a constant depending on the shear rate. The characteristic time of destructuration is $t_{c}=1 / B$, i.e. when $\exp (-B t)=e^{-1}$.

Several thixotropic models have been proposed to quantify the structural breakdown process of cementitious materials. Roussel has proposed models for cement paste [14]and concrete [15]. For fresh mortar under shear, the measured decay can be expected to be a function of both deflocculation of the colloidal suspension and sand particle migration. Concerning the latter, in arotational shear 
setup consisting of a concentrically oriented vane rotor and a cylindrical container, as the rotor introduces shearto a fresh mortar suspension sand particles will tend to migrate from the inner rotor to the wall of the cylinder.A void area with a smaller volume fraction will form in the suspension near the inner rotor, thus making the apparent viscosity and shear stress decrease. So modelling the shear stress decay provides a promising way to quantify the sand migration process.

Shear-induced particle migration has been explored in other system types, and the influence of different parameters has been measured by direct methods. It has been observed through magnetic resonance imaging (MRI) that shear-induced particle migration of beads in yield stress fluids is not apparent when the particle volume fraction is below $20 \%$, while it becomes more apparent at higher particle volume fractions [6].

Ovarlez et al. investigated the effect of shear rate on noncolloidal rigid particles suspended in a Newtonian fluid[16].They found very similar concentration profilesin a $58 \%$ suspension under constant rotational velocities ranging from 0.06 to $25 \mathrm{rpm}$ with preshears of 9-rpm and 100-rpm. At all rotational velocities with each preshear, the concentration was found to be lower near the inner cylinder where the shear rate is highest. Thismay be attributed to the concentration profile that is irreversibly established by the preshear. Ovarlez et al. [6]also found that the ratio of normal stress difference from the inner rotor to the wall, which is the force of shear-induced particle migration, is proportional to shear stress but not dependent on the shear rate.

In addition to particle volume fraction and shear rate, it is expected that mix constituents, namely the presence of chemical and mineral admixtures,will change the rheological properties of the suspending paste system and thereby change the kinetics of shear-induced sand particle migration. 
In this study, we propose a two exponential model to fit the torque/stress decayof fresh cement mortar systems subjected to constant angular velocity in a rotational rheometer. Results provide evidence that the colloidal and granular contributions to the overall destructuration and sand migration of mortars can be represented by each of the two exponentials.Model parameters are used to quantify the effect of sand volume fraction, clay addition, and applied shear rate on thekinetics and intensity of thixotropic deflocculation and particle migration. We find the influence of sand volume fraction androtational velocity on shear-induced particle migration, as described by the model parameters, agree well with the findings of other studies as presented in the literature review above.

\section{Experimental methods and materials}

\subsection{Materials}

All mixes are prepared with tap water and Type I Portland cement. According to ASTM C150[17], its compressive strength at 28 days is $44.8 \mathrm{MPa}$, the Blaine fineness is $420 \mathrm{~m}^{2} / \mathrm{kg}$ and the chemical constituents are summarized in Table 1. The sand used in this study is silica-quartz. It is oven-dried for 24 hours and sieved between sieve \#16 and \#30, yielding diameters between 0.6 and $1.18 \mathrm{~mm}$.

Highly purified attapulgite clay, or magnesium aluminosilicate, is also used. It isa commercially available clay that is chemically exfoliated from bulk attapulgite to remove all impurities. When dispersed, it is needle-like with an average length of $1.75 \mu \mathrm{m}$ and diameter of $3 \mathrm{~nm}$, which gives rise to a high aspect ratio and high specific surface area. Given its nanoscale dimension, it will be referred to as nanoclay herein. 
Table 1 Cement chemical constituents

\begin{tabular}{cc}
\hline Constituents & \% by mass \\
\hline $\mathrm{SiO}_{2}$ & 19.22 \\
$\mathrm{Al}_{2} \mathrm{O}_{3}$ & 4.98 \\
$\mathrm{Fe}_{2} \mathrm{O}_{3}$ & 3.42 \\
$\mathrm{CaO}$ & 62.42 \\
$\mathrm{MgO}$ & 3.87 \\
$\mathrm{SO}_{3}$ & 2.72 \\
\hline
\end{tabular}

\subsection{Mix proportion}

All mortar mixes have a water-to-cement $(\mathrm{w} / \mathrm{c})$ ratio of 0.5 by mass. Water absorption of the sand is considered when proportioning to achieve the desired $\mathrm{w} / \mathrm{c}$ ratio.

To explore the effect of sand volume, we test mortars with sand-to-cement (s/c) ratios of 1.5, 1.75, 2, and 2.25 , yielding sand volume fractions of $41 \%, 45 \%, 48 \%$, and $51 \%$, respectively. In this case, nanoclay addition is held constant at $0.5 \%$ by mass of cement, which is found to be sufficient to achieve stable mixes that exhibit no visible signs of static bleeding or sand sedimentation. To explore the effect of nanoclay, we test mortars with nanoclay additions of $0,0.25$ and $0.5 \%$ by mass of cement. In this case, s/c ratio is held constant at 2 by mass. Finally, to explore the effect of angular velocity all mixes have s/c ratio of 2 and $0.5 \%$ nanoclay addition.

\subsection{Mortar preparation}

In this study, steps are taken to ensure that the cement paste phase is mixed at the same state for various s/c ratios. First, only the fresh cement paste phase is prepared in a medium upright planetary 
mixer. Then, to ensure that the sand is mixed evenly in the cement paste, sand is poured into the cement paste and hand-mixed randomly at high intensity. The details of the protocol are given here.

Nanoclay powder is blended with the mixing water in a Waring blender for 2 minutes to produce a nanoclay suspension, which remains stable for at least 6 hours. The clay suspension and remaining mix water to reach the target $\mathrm{w} / \mathrm{c}$ ratio is poured into the mixing bowl. Cement powderis slowly poured into the wet ingredients and mixed at a speed of $136 \mathrm{rpm}$ for 1 minute, then at a higher speed of $281 \mathrm{rpm}$ for an additional 4 minutes. We occasionally scrape the bowl to ensure the cement paste is mixed evenly.Then, sand is slowly poured into the cement paste and hand-mixed in a random manner for 4 minutes. Finally the mortar is poured into the construction cell of the rheometer for testing.

\subsection{Rheometer and construction cell}

The rheometer in this study is a HAAKE MARS III rheometer. The construction cell is a $74 \mathrm{~mm}$ diameter and $150 \mathrm{~mm}$ height cylinder, with 24 profiles of $2 \mathrm{~mm}$ evenly distributed at the wall to prevent wall slip. The rotor is a two-bladed vane with a diameter of $52 \mathrm{~mm}$ and height of $50 \mathrm{~mm}$. The coaxial gapbetween rotor and construction cell is $11 \mathrm{~mm}$, which is much larger than the size of sand. For all the tests in this study, the vane rotor is placed at the height where the gap between the bottom of the rotor and bottom of the construction cell is $20 \mathrm{~mm}$.

\subsection{Protocol}

As cementitious materials are thixotropic, the flow behavior is greatly dependent on the flocculation state. Therefore it must be consistent between each test to ensure reasonable repeatability. Since the 
completely de-flocculated state is theoretically impossible to reach, Roussel [15] recommends using the "most de-flocculated state" as the reference state in studying thixotropic materials. He also states that the most de-flocculated state is achieved right after mixing, since rheometers do not reach the high mixing speed mixers do. Since this state can only be reached immediately after mixing, we have adopted an alternative reference state based on the work of Mahaut et. al. [18], which is to introduce random mixing by hand after the mortar sample is loaded in the rheometer. The advantage of random hand mixing over applying a preshear with the rotor is that it preventspreferential orientation in the shear direction of the planetary mixerand facilitates a random homogeneous mix of sand in cement paste. Therefore in this study, before each test the mortars are strongly tampered by hand mixing for 1 minute in a random manner to bring the material to a homogeneous state.

Another point that should be considered is irreversible workability loss due to cement hydration. At the sample age we are investigating, within 1 hour of initial water to cement contact, the characteristic time of irreversible workability loss due to cement hydration can be expected to be much longer than that of paste deflocculation and sand migration. Jarny, Roussel et al. [19] found that within shorter time periods thixotropic effects dominate, while at longer periods the irreversible effects of cement hydration dominate. Struble et al. $[20,21]$ also show that before initial setting (around 1.5 hours after water addition), the irreversible effects of hydration are marginal and the yield stress increases very slowly with resting time. In this study, test results of constant rotational velocityalso show that the difference in equilibrium torque is less than $3 \%$ within this time period. Therefore it is assumed that the irreversible effects of hydration are negligible and random strong hand mixing canbring the mortar into a replicate deflocculatedstate. 
Immediately after 10 minutes mixing, the fresh mortar is poured into the construction cell. Before each test, the mortar is hand tampered for 1 minute using a standardwhisk. Then the rotor is quickly put in position and lowered to the designated position. The shearing protocol used in this study is simple: constant angular velocity for 60 seconds. The torque is recorded at a data acquisition rate of 4 data points per second at $10 \mathrm{rad} / \mathrm{s}$, and 8 data points per second at higher shear rate. With the vane geometry used in this study, effective shear rate and shear stress are not easy to derive. Therefore results will be reported in angular velocity of rotor $(\mathrm{rad} / \mathrm{s})$ and shear torque $(\mathrm{mNm})$. At least three samples per mix are tested and the average is taken to be the representative value. Errors bars are presented in all plots to show variability.

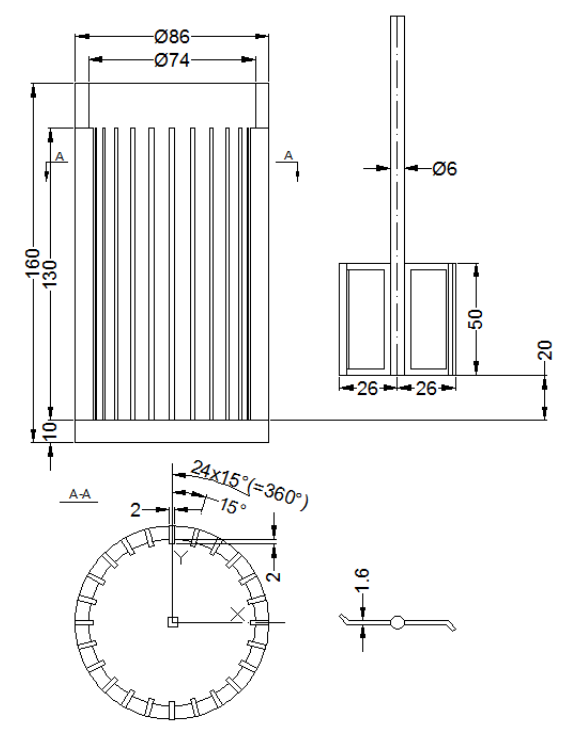

Figure 1Dimensions of construction cell and rotor.

\section{Results and discussion}

3.1 A modified thixotropy model for mortar 
A number of thixotropy models exist, reviewed by Roussel [15], to describe flocculation and deflocculation behavior of cement-based materials. Although described in detail elsewhere, it will be briefly presented here.

The general form for existing models can be written as follows:

$$
\tau=(1+\lambda) \tau_{0}+k \dot{\gamma}^{n} \quad \text { Eq. } 2
$$

$$
\frac{\partial \lambda}{\partial t}=\frac{1}{T \lambda^{m}}-\alpha \lambda \dot{\gamma}
$$

Where $\lambda$ is the flocculation state of the material and $\mathrm{T}, \mathrm{m}$, and $\alpha$ are thixotropy parameters. Two assumptions are applied: i) the steady-state flow can be described by the Bingham model and ii) the yield stress at rest increases linearly. It is reasonable to assume both for our cement mortars systems based on the results of other studies on concretes [15, 22] and mortars [23].

From there a simplified deflocculation model is found to be:

$$
\tau=\left(1+\lambda_{0} e^{-\alpha \dot{\gamma} t}\right) \tau_{0}+\mu_{p} \dot{\gamma}
$$

Eq. 4

where $\mu_{\mathrm{p}}$ is plastic viscosity.

It can be noted that the model predicts, just as the Tattersall model [11] and Papo model [12], an exponential decrease of the shear stress under constant shear rate with a deflocculation characteristic time equal to $1 /(\alpha \dot{\gamma})$. 
It has been found that shear stress decay under shear of pastes and concretes can be described by a single exponential $[14,15,22]$. However, we find that the stress decayof mortars cannot, especially at the initial onset of shear. And given the rapid kinetics of shear-induced particle migration, it is essential to capture the initial portion of the decay. Instead, it requires two exponentials, as shown in Figure 2. It is hypothesized that it has to do with the viscous and granular contributions in mortar systems that is not as present in cement paste systems. Thus it is proposed that the stress decayof mortars under shear be described by the following:

$$
M=M_{0}+M_{1} e^{-\alpha_{1} \dot{\gamma} t}+M_{2} e^{-\alpha_{2} \dot{\gamma} t}
$$

Eq. 5

where the colloidal deflocculation of the paste phaseand sand shear migrationof the granular phase (i.e. sand particles) are described by each exponential.

The characteristic time of each component is given by $1 /\left(\alpha_{1} \dot{\gamma}\right)$ and $1 /\left(\alpha_{2} \dot{\gamma}\right)$. The shorter the characteristic time, which corresponds to the biggerovalue at a given shear rate, the faster the rate ofstress decay.Of the three torque parameters, $\mathrm{M}_{0}$ corresponds to the steady state of the mortar systemunder constant shear,while $\mathrm{M}_{1}$ and $\mathrm{M}_{2}$ correspond to the intensity of colloidal deflocculation and sand migration, respectively.We observe the effect of s/c ratio, nanoclay addition and angular velocityon the parameters to explore the suitability of the model for distinguishing and characterizing colloidal deflocculation and sand shear migration during transient flow of fresh mortar. 


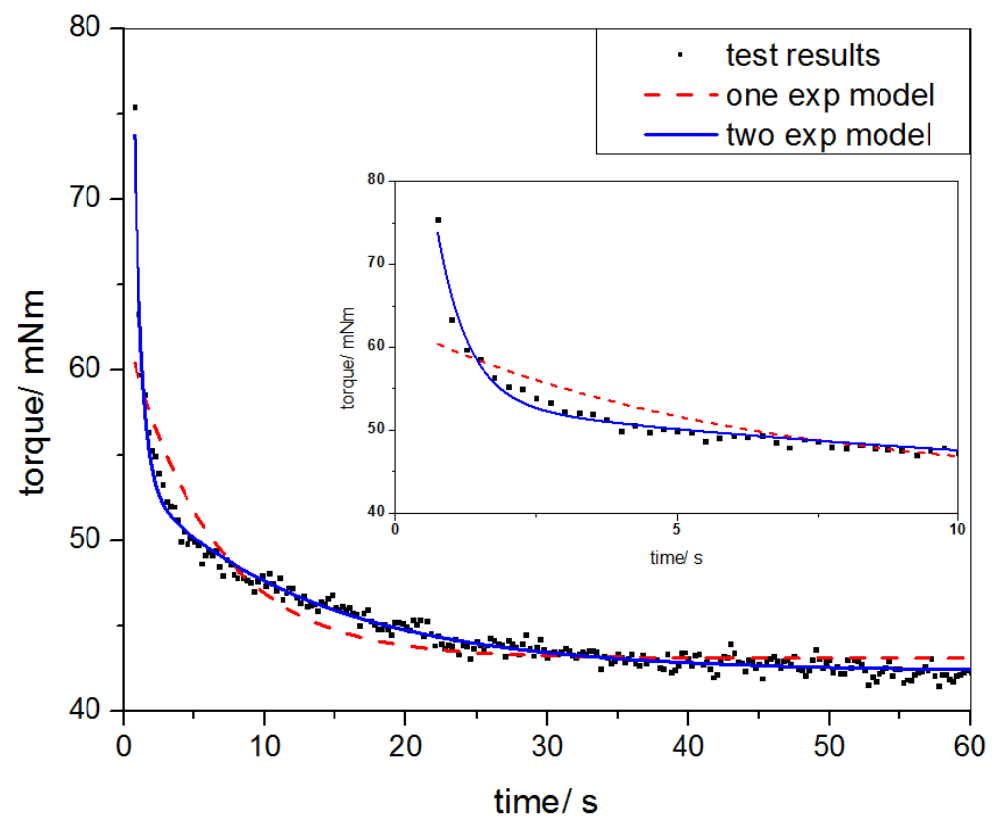

Figure 2One versus two exponential model for capturing stress decay of fresh mortar $(\mathrm{s} / \mathrm{c}=2)$ under constant angular velocity (10 rad/s).

\subsection{Effect of $\mathrm{s} / \mathrm{c}$ ratio}

We capture the stressdecay of fresh mortars with s/c ratios ranging between 1.50 and 2.25 at a constant applied rotational rate of $10 \mathrm{rad} / \mathrm{s}$ over 60 seconds. All the samples have $0.5 \%$ nanoclay addition by mass of cement. The results of parameters aandM are presented in Figures 3 and 4, respectively. First, with increasing s/c ratio all $\alpha$ and $M$ parameters increase, indicating faster and higher degree of stress decayoverall. Second, comparing $\alpha_{1}$ and $\alpha_{2}, \alpha_{1}$ is about 20 times greater than $\alpha_{2}$ throughout while the difference between $\mathrm{M}_{1}$ and $\mathrm{M}_{2}$ is much smaller. This indicates there are two distinct stress decaymechanisms at different rates yet similar intensities. Third, it is apparent that there is greater variability for $\alpha_{1}$, especially for $\mathrm{s} / \mathrm{c}=2.25$. This variability can be expected as it describes the instantaneous decay upon introduction of shear, which highly depends on the initial flocculation state. 
Focusing on rate of decay, we observe an increase in $\alpha_{1}$ and $\alpha_{2}$ with increase in $\mathrm{s} / \mathrm{c}$ ratio, which translates to decrease in characteristic times $1 / \alpha_{1} \dot{\gamma}$ and $1 / \alpha_{2} \dot{\gamma}$. This can be attributed to accelerated kinetics of both colloidal deflocculation of the paste phase and shear migration of the sand. Increase in sand volume fraction will increase the local shear rate applied on the paste phase, thusacceleratingcolloidal deflocculation andcorresponding rate of decay. And as mentioned prior, concentration profiles of particles measured through MRI showedshear-induced particle migration increases with particle volume fraction[6]. Further, it could be reasoned that $\alpha_{1}$ and $\alpha_{2}$ correspond to paste deflocculation and sand migration, respectively.

First, due to the fine size of cement particles compared with sands, the characteristic strain to break down the paste colloidal structure is much smaller than that of sand migration. So at constant shear rate, the characteristic time for colloidal deflocculation is much smaller than that of sand migration. This means the parameteracorresponding to colloidal deflocculation can be expected to be much greater, which points to $\alpha_{1}$. Secondly, with increasing s/c from 1.5 to $2.25, \alpha_{2}$ increases more timesthan $\alpha_{1}$, indicating that $\alpha_{2}$ is more sensitive to sand volume fraction. Through MRI, Ovarlez et al.[6] found that particle migration became more apparent as particle volume fractions exceeded 30\% and approached the maximum packing density (around 64\% for monodisperse spheres). In our present study, s/c ratios 1.5, 1.75, 2 and 2.25 correspond to sand volume fractions $41 \%, 45 \%, 48 \%$, and $51 \%$, respectively. Therefore in this range we can expect that sand volume fraction will have a greater effect on sand migration over deflocculation of cement paste, thus tying sand migration to $\alpha_{1}$. 


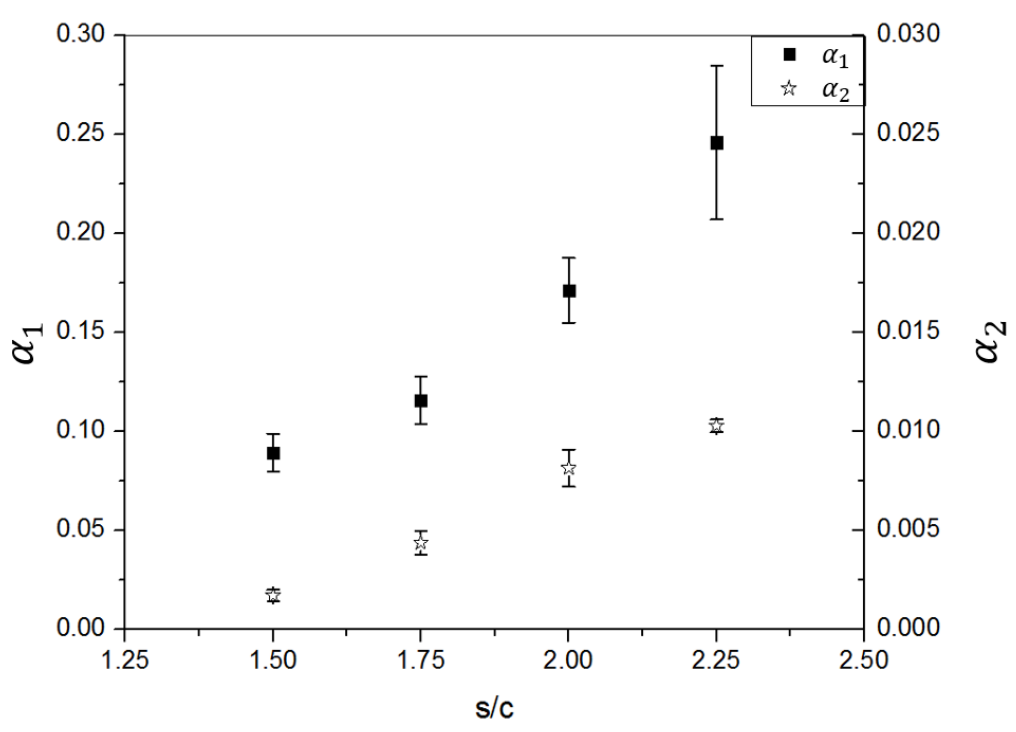

Figure 3Effect of s/c ratio on parameters $\alpha_{1}$ and $\alpha_{2}$.

FromFigure 4 (a), all the torqueM values increase with s/c ratios, indicatinga denser steady-state structure and higher degree of decay. To observe the effect of s/c ratio on the relative intensity of paste deflocculation and sand migration, we compare the relative increase of each torque by normalizing $\mathrm{M}_{\mathrm{x}}$, as follows:

$$
\text { Normalized } M_{x}=M_{x} /\left(M_{0}+M_{1}+M_{2}\right)
$$

Eq. 6

where $M_{0}+M_{1}+M_{2}$ corresponds to the maximum value of stress under constant shear rate. Normalized $\mathrm{M}_{0}$ corresponds to the ratio between viscosity before and aftercolloidal deflocculation and sand migration, which translates to the ratio between dynamic yield stress and static yield stress;normalized $\mathrm{M}_{1}$ and $\mathrm{M}_{2}$ correspond to the relative intensity of each component over the maximum torque. From Figure 4 (b), we observe that normalized $M_{1}$ and $M_{2}$ increase withsand volume, indicatinghigher degree of colloidal deflocculation and sand migration during the shearing period. This is, again, in agreement with the expected effect of increasing sand volume fraction. However, normalized $\mathrm{M}_{0}$ decreases, indicating sand addition increases static yield stress more than dynamic yield stress.Measuring static yield stress through the stress growth protocol, Mahaut et al. [18] found that the static yield stress increases with particle volume fraction, where the increment of 
increase is higher at higherparticle volume fraction. However, $\mathrm{Hu}$ [24] found that the dynamic yield stress of mortar increases less at higher sand volume fraction. These results support that normalized $\mathrm{M}_{0}$, as the ratio between dynamic yield stress and static yield stress, decreases with increasing sand volume fraction.

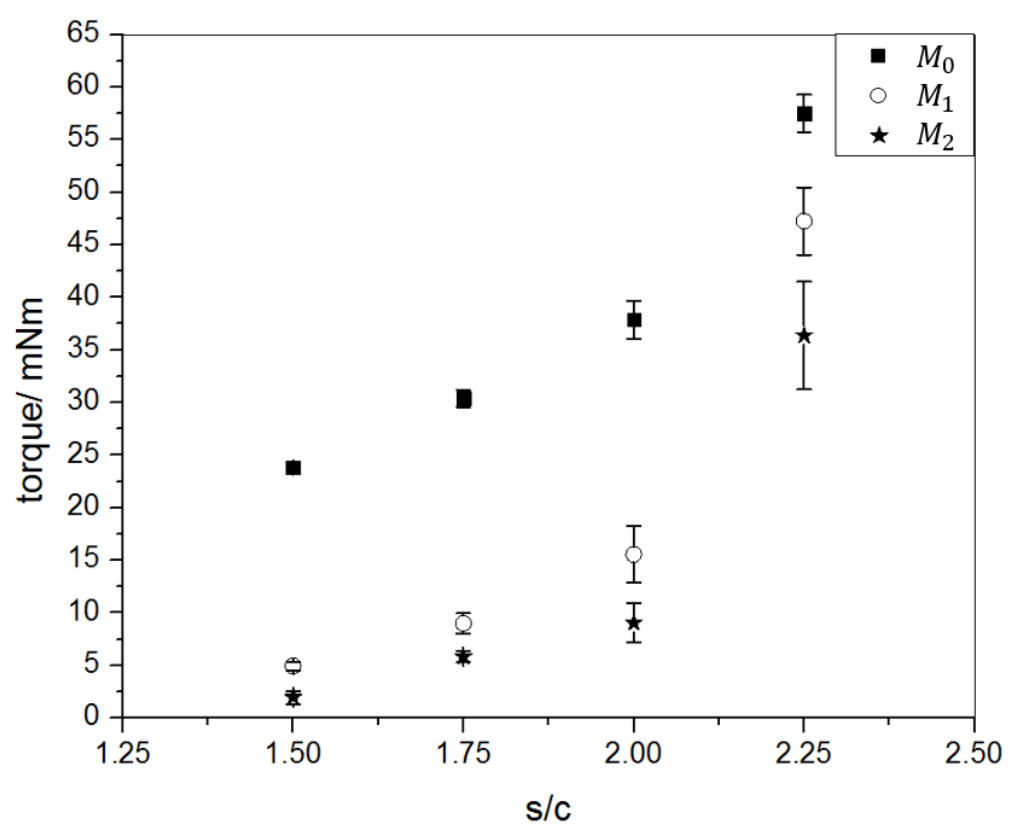

(a)

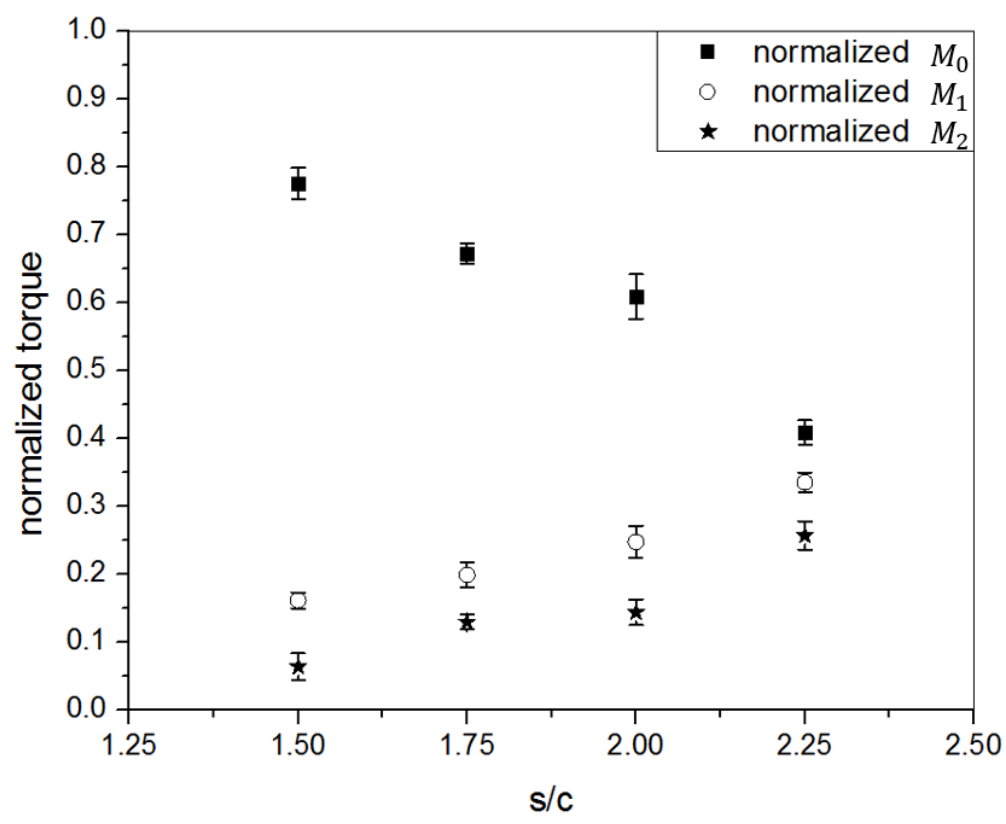


(b)

Figure 4Effect of s/c ratio on (a) parameters $\mathrm{M}_{0}, \mathrm{M}_{1}$, and $\mathrm{M}_{2}$ and (b) normalized $\mathrm{M}_{0}, \mathrm{M}_{1}$, and $\mathrm{M}_{2}$.

\subsection{Effect of clay addition}

The effect of nanoclay addition was investigated on mortar systems with s/c ratio 2 subjected to an angular velocity of $10 \mathrm{rad} / \mathrm{s}$. The nanoclay addition varies from 0 to $0.5 \%$ by mass of cement. The results of aand M parameters are presented in Figures 5 and 6, respectively.

Firstly looking at $\alpha$ parameters(Figure 5),ataddition levels up to $0.5 \%$ nanoclay by mass of cementthere is an apparent increase in $\alpha_{1}$ and decrease in $\alpha_{2}$. In this model, $1 / \alpha_{1} \dot{\gamma}$ corresponds to the characteristic time of deflocculation of the paste phase - thus, $1 / \alpha_{1}$ corresponds to the characteristic strain for deflocculation. Since $\dot{\gamma}$ here is constant, increase in $\alpha_{1}$ leads todecrease in characteristic strain. Physically, this indicates that the nanoclays are stiffening the microstructure of the cement paste.In a parallel investigation we implemented low amplitude oscillatory shear (LAOS) to measure the critical strainat which the fresh-state structureof fresh cement pastes modified with nanoclays is irreversibly broken, the results of which will be fully reported in another paper[25]. Details of the method can be found elsewhere [26], although briefly explained here. Within the linear viscoelastic region (LVR), the storage modulus G' and loss modulus G” are independent of the applied strain amplitudebecause the applied oscillatory strain is sufficiently small and the suspension structure remains intact. Beyond a critical strain, which marks the end of the LVR, the microstructure can no longer fully recoverand G' dramatically decreases. To measure the critical strain, we apply a strain amplitude sweep from $10^{-5}$ to $10^{-1}$ at a frequency of $1 \mathrm{~Hz}$, as conducted in many studies on cement paste [26-28]. Critical strain values of clay-modified cement pastes are reported in Table 2. It is 
apparent that critical strain decreases with nanoclay addition, which supports that $\alpha_{1}$ corresponds tothe paste phase.

The decrease in $\alpha_{2}$ indicates the rate of sand migration is slowedwith nanoclay addition. Viscosity modifiersare commonly used to improve suspension stability, particularly in SCC mixes. For instance, a number of studies have demonstrated that viscosity modifying agents (VMAs) can improve dynamic segregation resistance[29-31].Clays can have a similar effect. Metakaolin clay addition in cementhas been found to stabilize pastes, especially at prolonged mixing or higher w/b ratio [32]. And nanoclays have been shown to increase yield stress [33], apparent viscosity[34] and cohesiveness[35] of cement paste at modest dosages. Based on the results of the references studies it can be expected that nanoclays can slow particle migration, which supports that $\alpha_{2}$ corresponds to the granular phase.

As for the intensity parameters, $\mathrm{M}_{0}$ and $\mathrm{M}_{1}$ increase with nanoclay addition, which can be attributed to the increase in viscosity of the overall system and paste phase, respectively[33-35]. On the other hand, $\mathrm{M}_{2}$ remains relatively constantat all nanoclay addition levels. From Figure 6 (b), we observe that normalized $\mathrm{M}_{0}$ increases with nanoclay addition while normalized $\mathrm{M}_{2}$ decreases. Increase in normalized $\mathrm{M}_{0}$ captures the significant flocculating effect of the clays, which has been observed in other studies. Through focused beam reflectance measurement (FBRM), Ferron et al. [36] found that various clay types increase average floc size. And through compressive rheology, Tregger et al. [37] found that nanoclays increased floc strength. The decrease in normalized $\mathrm{M}_{2}$ indicates that the flocculating effect of the clays on the pastes phase subsequently leads to some alleviation of sand migration. 


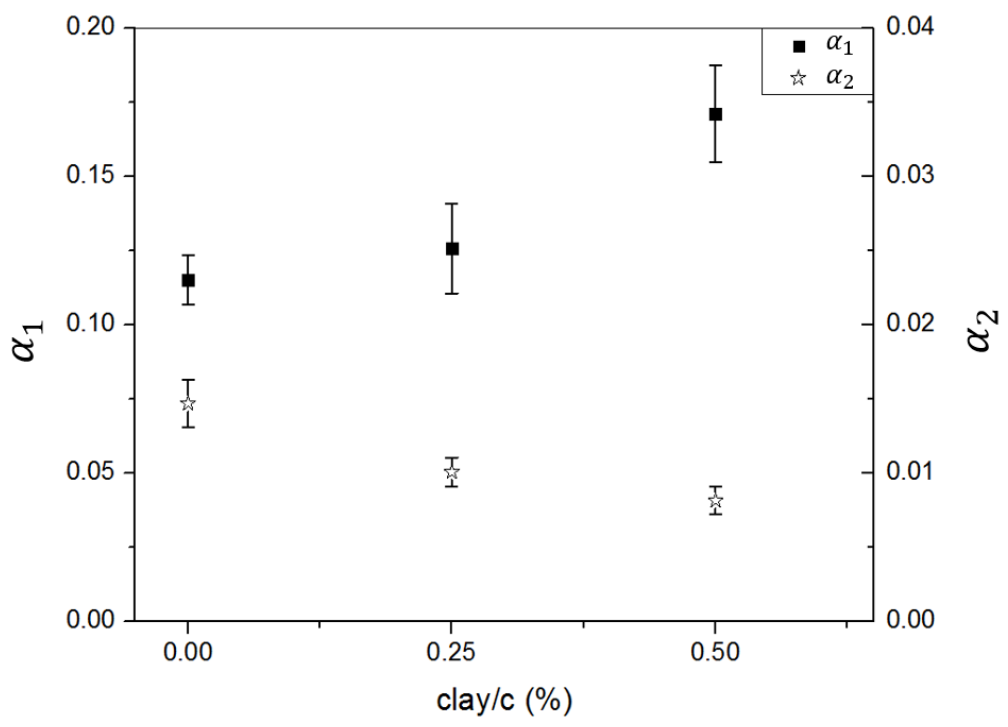

Figure 5Effect of nanoclay addition on parameters $\alpha_{1}$ and $\alpha_{2}$.

Table 2 Effects of nanoclay addition on critical strain

\begin{tabular}{ccc}
\hline Nanoclay addition over cement/ \% & Average critical strain & Standard deviation \\
\hline 0 & $7.69 \mathrm{E}-4$ & $5.1 \mathrm{E}-5$ \\
0.1 & $3.69 \mathrm{E}-4$ & $3.2 \mathrm{E}-5$ \\
0.3 & $2.42 \mathrm{E}-4$ & $1.9 \mathrm{E}-5$ \\
0.5 & $1.69 \mathrm{E}-4$ & $5.6 \mathrm{E}-5$ \\
\hline
\end{tabular}




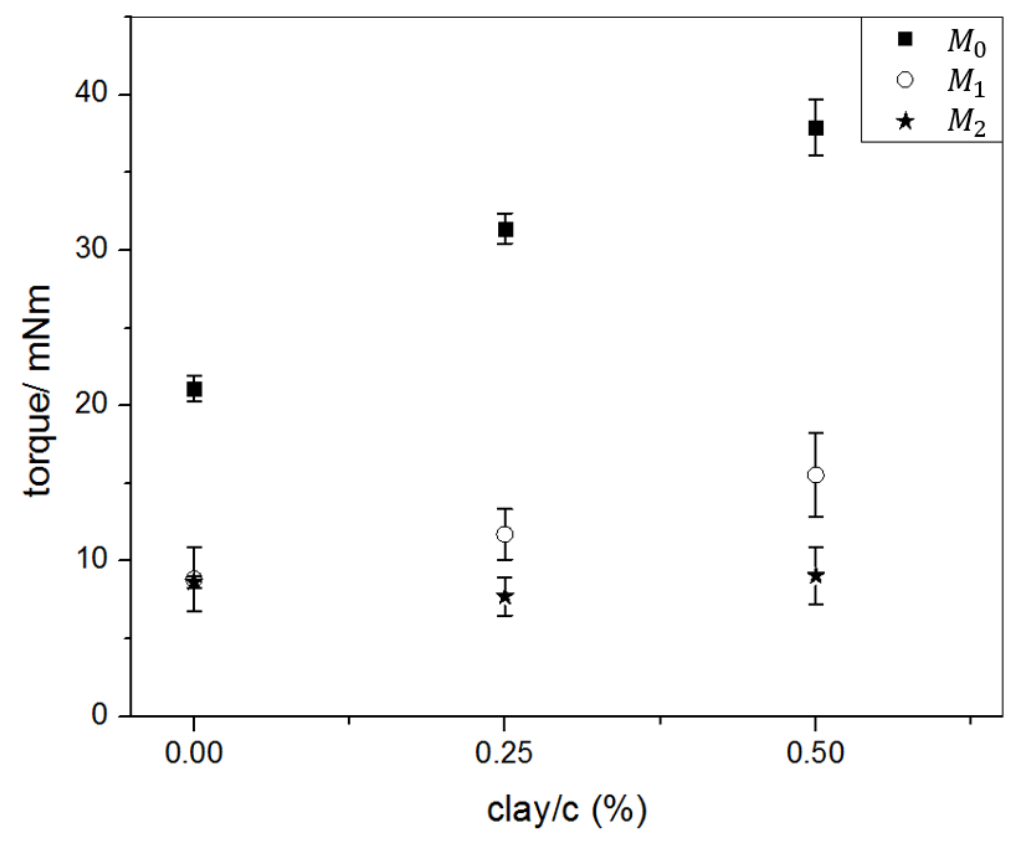

(a)

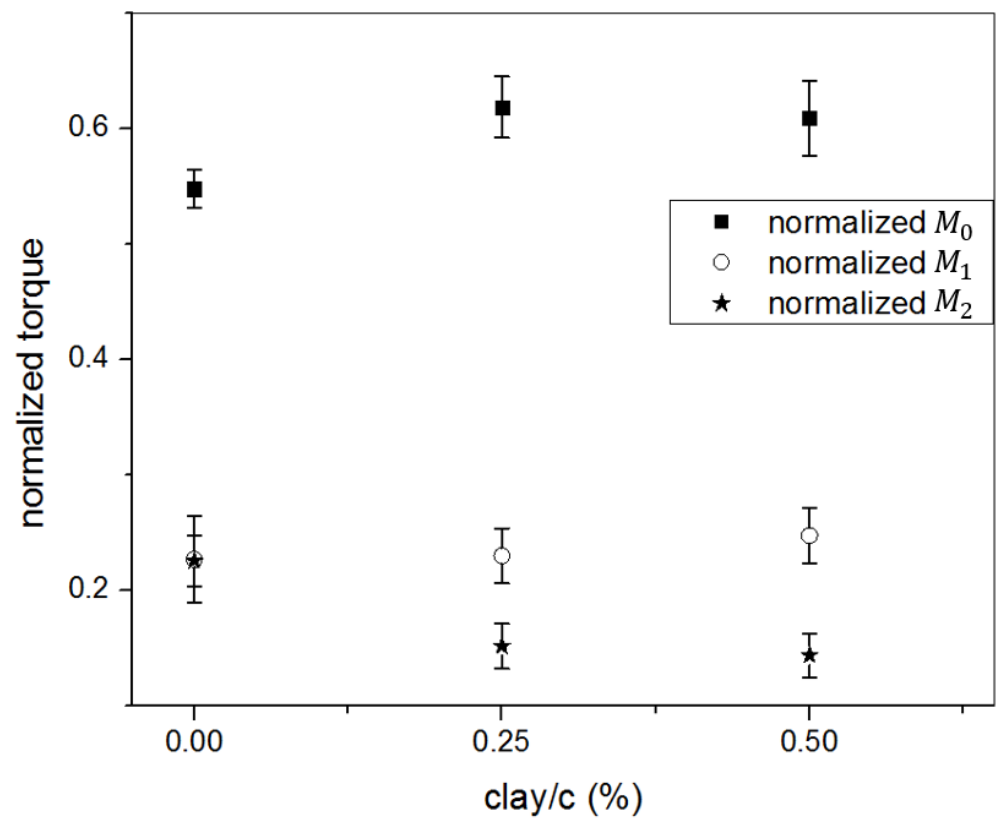

(b)

Figure 6Effect of nanoclay addition on (a) parameters $\mathrm{M}_{0}, \mathrm{M}_{1}$, and $\mathrm{M}_{2}$ and (b) normalized $\mathrm{M}_{0}$, $\mathrm{M}_{1}$, and $\mathrm{M}_{2}$.

\subsection{Effect of angular velocity}


The effect of angular velocity on $\alpha \dot{\gamma}$ and $\mathrm{M}$ parameters are shown in Figure 7 and 8, respectively. Overall, changes in angular velocity in the range of $10 \mathrm{rad} / \mathrm{s}$ to $20 \mathrm{rad} / \mathrm{sshow}$ less influence on the parameters of the model compared to sand volume fraction and nanoclay addition.

Looking at $\alpha \dot{\gamma}$ parameters (Figure 7), $\alpha_{1} \dot{\gamma}$ remains relatively constant within $20 \mathrm{rad} / \mathrm{s}$. This supports that $10 \mathrm{rad} / \mathrm{s}$ is sufficiently high to break the colloidal structure, which is the applied angular velocity implemented throughout the investigation. $\alpha_{2} \dot{\gamma}$ shows slight increase with higher angular velocity this corresponds to lower characteristic time $1 / \alpha_{2} \dot{\gamma}$, indicating faster kinetics of sand migration.From Figure 8 (a), there is no apparent trend of the M parameters with angular velocity. It is expected that increase in angular velocity will increase viscosity of the overall system. Here, $\mathrm{M}_{0}$ shows a slight increase with angular velocity but it is not apparent. Looking at the effect of angular velocity on normalized M(Figure 8 (b))provides more insight. All normalized $\mathrm{M}$ values remain relatively constant with angular velocity. Specifically,normalized $\mathrm{M}_{2}$ does not change. $\mathrm{M}_{2}$ does not change either (Figure 8 (a)). This shows that although angular velocity can accelerate kinetics (i.e. increase in $\alpha_{2} \dot{\gamma}$ ), there is no measurable increase in intensity of sand migration within the total shearing period.This canat least partially explain why $\mathrm{M}_{\mathrm{o}}$ does not show an apparent increase with shear rate degree of sand migration is similar at steady-state. This is in agreement with the observations of Ovarlez et al. $[6,16]$, where similar concentration profiles were captured at a range of pre-shear and applied constant shear rate conditions. 


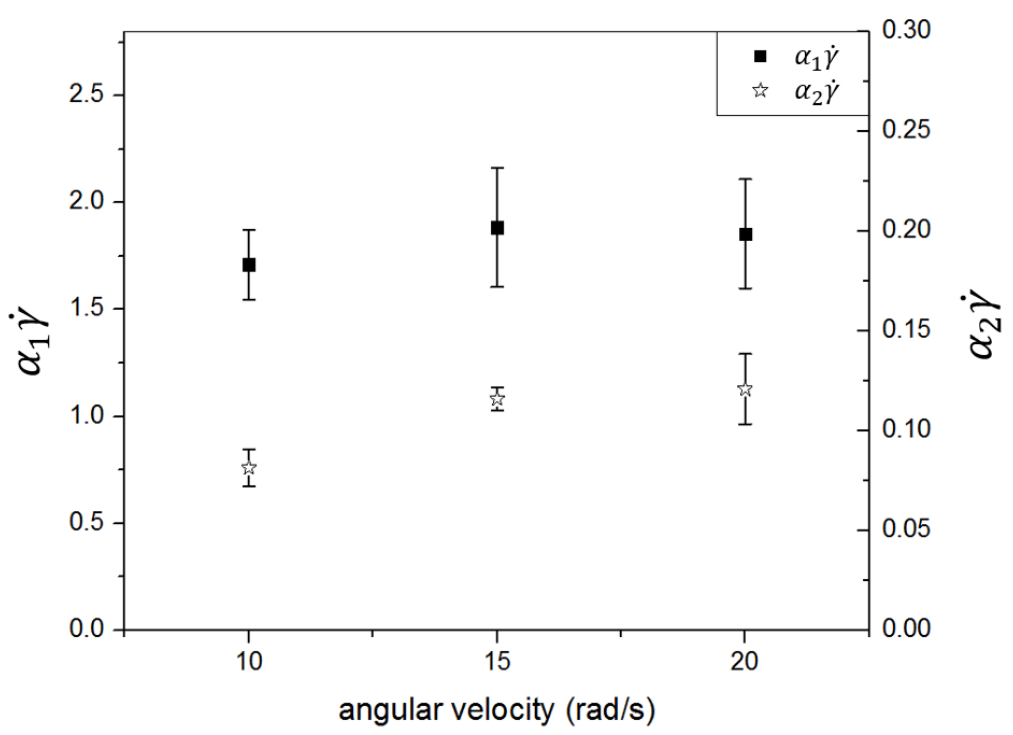

Figure 7Effect of angular velocity on parameters $\alpha_{1} \dot{\gamma}$ and $\alpha_{2} \dot{\gamma}$.

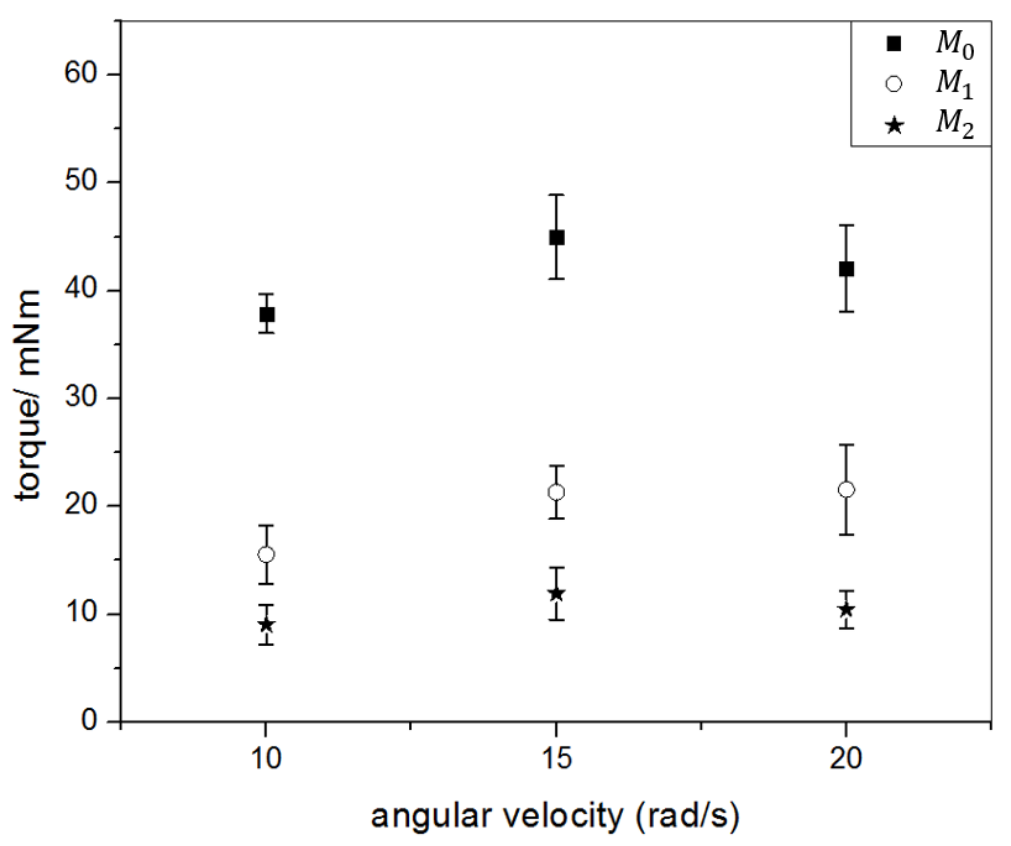

(a) 


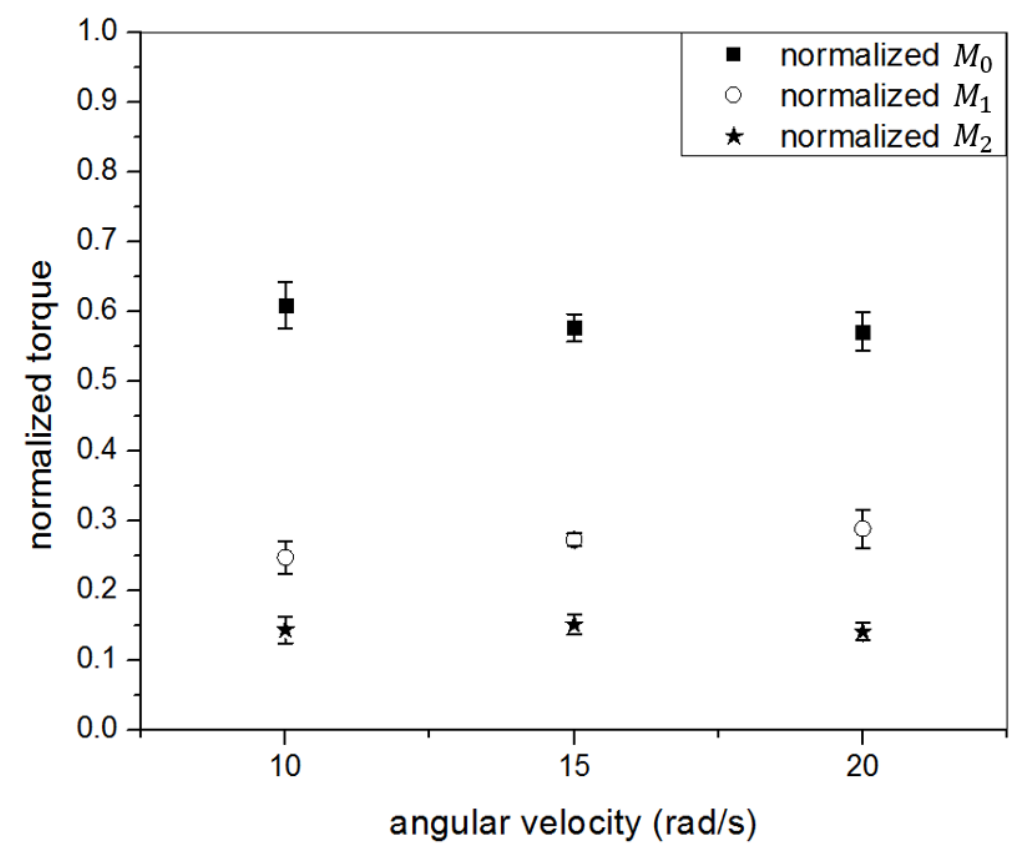

(b)

Figure 8Effect of angular velocity on (a) parameters $\mathrm{M}_{0}, \mathrm{M}_{1}$, and $\mathrm{M}_{2}$ and (b) normalized $\mathrm{M}_{0}, \mathrm{M}_{1}$, and $\mathrm{M}_{2}$.

\section{Conclusions}

In this study, a modified two exponentialthixotropy/migration model is proposed to quantify the stress decay of fresh mortar under constant shear rate. Results support that destructuration and sand migration are represented by the two exponentials. $\alpha$ and characteristic time $1 /(\alpha \dot{\gamma})$ are related to the rate of these two stress decaymechanisms, and torque $\mathrm{M}$ and normalized $\mathrm{M}$ indicate the intensity of each phase. Test results indicate the following:

- Increase of sand volume fraction increases all five parameters,along with normalized $\mathrm{M}_{1}$ and $\mathrm{M}_{2}$, indicating faster kinetics and increased intensity of both colloidal deflocculation and sand migration. This is in agreement with observations of other studies. 
- Nanoclay addition increases $\alpha_{1}$ and decreases $\alpha_{2}$, indicating decrease in critical strain, which agrees well withLAOSresults, and slowed sand migration. Decrease of normalized $\mathrm{M}_{2}$ supports the alleviation of sand migration with nanoclay addition.

- Angular velocity in the range of 10 to $20 \mathrm{rad} / \mathrm{s}$ does not have a significant influence on the model parameters. Increase in angular velocity increases $\alpha_{2}$ slightly, indicating slightly accelerated shear migration kinetics. However, intensity of sand migration, $\mathrm{M}_{2}$ and normalized $\mathrm{M}_{2}$, is not greatly affected by angular velocity, which agrees well with results in literature.

The thixotropy/migration model provides a straightforward and promisingway to measure the sand migration behavior of mortar. Future work includes exploring other parameters (e.g. rheometer geometry, mix design, increased range of angular velocities), as well as supporting flow results with direct measurements of concentration profile to help validate the model.

\section{Acknowledgements}

The authors would like to acknowledge the National Science Foundation (NSF EEC-1342377) and Columbia University's Office of the Provost's Grants Program for financial support, and technical support by the staff of Columbia University's Carleton Laboratory.Also, the authors would like to thank Essex Cement LLC and Active Minerals for providing materials. 


\section{References}

[1] E.P. Koehler, D.W. Fowler, C. Ferraris, Summary of concrete workability test methods, 2003.

[2] C.F. Ferraris, L.E. Brower, P. Banfill, Comparison of concrete rheometers: International tests at LCPC (Nantes, France) in October, 2000, US Department of Commerce, National Institute of Standards and Technology2001.

[3] D. Beaupré, F. Chapdelaine, P. Domone, E. Koehler, L. Shen, M. Sonebi, L. Struble, D. Tepke, J. Wallevik, O. Wallevik, Comparison of concrete rheometers: International tests at MBT (Cleveland OH, USA) in May 2003, (2004).

[4] Z. Toutou, N. Roussel, Multi scale experimental study of concrete rheology: from water scale to gravel scale, Mater Struct, 39 (2006) 189-199.

[5] EFNARC, Specification and guidelines for self-compacting concrete, Famham, Surrey GU9 7EN, UK, website: www.efnarc.org, ISBN 953973344, February 2002.

[6] G. Ovarlez, F. Mahaut, S. Deboeuf, N. Lenoir, S. Hormozi, X. Chateau, Flows of suspensions of particles in yield stress fluids, Journal of Rheology (1978-present), 59 (2015) 1449-1486.

[7] H. Hafid, G. Ovarlez, F. Toussaint, P. Jezequel, N. Roussel, Assessment of potential concrete and mortar rheometry artifacts using magnetic resonance imaging, Cement and Concrete Research, 71 (2015) 29-35.

[8] O.H. Wallevik, D. Feys, J.E. Wallevik, K.H. Khayat, Avoiding inaccurate interpretations of rheological measurements for cement-based materials, Cement and Concrete Research, (2015).

[9] J. Spangenberg, N. Roussel, J. Hattel, H. Stang, J. Skocek, M. Geiker, Flow induced particle migration in fresh concrete: Theoretical frame, numerical simulations and experimental results on model fluids, Cement and Concrete Research, 42 (2012) 633-641.

[10] N. Roussel, G. Ovarlez, S. Garrault, C. Brumaud, The origins of thixotropy of fresh cement pastes, Cement and Concrete Research, 42 (2012) 148-157. 
[11] G. Tattersall, The rheology of Portland cement pastes, British Journal of Applied Physics, 6 (1955) 165.

[12] A. Papo, The thixotropic behavior of white portland cement pastes, Cement and Concrete Research, 18 (1988) 595-603.

[13] R. Lapasin, V. Longo, S. Rajgelj, Thixotropic behaviour of cement pastes, Cement and Concrete Research, 9 (1979) 309-318.

[14] N. Roussel, Steady and transient flow behaviour of fresh cement pastes, Cement and concrete research, 35 (2005) 1656-1664.

[15] N. Roussel, A thixotropy model for fresh fluid concretes: theory, validation and applications, Cement and Concrete Research, 36 (2006) 1797-1806.

[16] G. Ovarlez, F. Bertrand, S. Rodts, Local determination of the constitutive law of a dense suspension of noncolloidal particles through magnetic resonance imaging, Journal of Rheology (1978-present), 50 (2006) 259-292.

[17] A. C150, Standard Specification for Portland Cement, ASTM International, West Conshohocken, PA, 2015.

[18] F. Mahaut, S. Mokeddem, X. Chateau, N. Roussel, G. Ovarlez, Effect of coarse particle volume fraction on the yield stress and thixotropy of cementitious materials, Cement and concrete research, 38 (2008) 1276-1285.

[19] S. Jarny, N. Roussel, R. Le Roy, P. Coussot, Modelling thixotropic behavior of fresh cement pastes from MRI measurements, Cement and Concrete Research, 38 (2008) 616-623.

[20] L.J. Struble, W.-G. Lei, Rheological changes associated with setting of cement paste, Advanced Cement Based Materials, 2 (1995) 224-230.

[21] W.G. Lei, L.J. Struble, Microstructure and flow behavior of fresh cement paste, J Am Ceram Soc, 80 (1997) 2021-2028.

[22] J. Assaad, K.H. Khayat, H. Mesbah, Assessment of thixotropy of flowable and selfconsolidating concrete, ACI Materials Journal, 100 (2003). 
[23] J. Assaad, K.H. Khayat, Assessment of Thixotropy of Self-Consolidating Concrete and Concrete-Equivalent-Mortar- Effect of Binder Composition and Content, ACI Materials Journal, 101 (2004).

[24] J. Hu, A study of effects of aggregate on concrete rheology, PhD Thesis, Iowa State University, Ames, Iowa, (2005).

[25] Y. Qian, S. Kawashima, Use of creep recovery protocol to measure static yield stress and structural rebuilding of fresh cement pastes, Cement and Concrete Research, (Under review).

[26] M.A. Schultz, L.J. Struble, Use of oscillatory shear to study flow behavior of fresh cement paste, Cement and Concrete Research, 23 (1993) 273-282.

[27] L. Nachbaur, J. Mutin, A. Nonat, L. Choplin, Dynamic mode rheology of cement and tricalcium silicate pastes from mixing to setting, Cement and Concrete Research, 31 (2001) 183-192.

[28] A. Betioli, P. Gleize, D. Silva, V. John, R. Pileggi, Effect of HMEC on the consolidation of cement pastes: Isothermal calorimetry versus oscillatory rheometry, Cement and concrete Research, 39 (2009) 440-445.

[29] K. Khayat, Workability, testing, and performance of self-consolidating concrete, ACI Materials Journal, 96 (1999).

[30] N.A. Libre, R. Khoshnazar, M. Shekarchi, Relationship between fluidity and stability of selfconsolidating mortar incorporating chemical and mineral admixtures, Construction and Building Materials, 24 (2010) 1262-1271.

[31] N. Tregger, A. Gregori, L. Ferrara, S. Shah, Correlating dynamic segregation of selfconsolidating concrete to the slump-flow test, Construction and Building Materials, 28 (2012) 499505.

[32] I. Mehdipour, M.S. Razzaghi, K. Amini, M. Shekarchi, Effect of mineral admixtures on fluidity and stability of self-consolidating mortar subjected to prolonged mixing time, Construction and Building Materials, 40 (2013) 1029-1037. 
[33] S. Kawashima, J.H. Kim, D.J. Corr, S.P. Shah, Study of the mechanisms underlying the freshstate response of cementitious materials modified with nanoclays, Construction and Building Materials, 36 (2012) 749-757.

[34] S. Kawashima, M. Chaouche, D.J. Corr, S.P. Shah, Rate of thixotropic rebuilding of cement pastes modified with highly purified attapulgite clays, Cement and Concrete Research, 53 (2013) 112-118.

[35] S. Kawashima, M. Chaouche, D.J. Corr, S.P. Shah, Influence of purified attapulgite clays on the adhesive properties of cement pastes as measured by the tack test, Cement and Concrete Composites, (2014).

[36] R.D. Ferron, S. Shah, E. Fuente, C. Negro, Aggregation and breakage kinetics of fresh cement paste, Cement and Concrete Research, 50 (2013) 1-10.

[37] N.A. Tregger, M.E. Pakula, S.P. Shah, Influence of clays on the rheology of cement pastes, Cement and concrete research, 40 (2010) 384-391. 


\section{List of Tables}

Table 1 Cement chemical constituents

\begin{tabular}{cc}
\hline Constituents & \% by mass \\
\hline $\mathrm{SiO}_{2}$ & 19.22 \\
$\mathrm{Al}_{2} \mathrm{O}_{3}$ & 4.98 \\
$\mathrm{Fe}_{2} \mathrm{O}_{3}$ & 3.42 \\
$\mathrm{CaO}$ & 62.42 \\
$\mathrm{MgO}$ & 3.87 \\
$\mathrm{SO}_{3}$ & 2.72 \\
\hline
\end{tabular}

Table 2 Effects of nanoclay addition on critical strain

\begin{tabular}{ccc}
\hline Nanoclay addition over cement $/ \%$ & Average critical strain & Standard deviation \\
\hline 0 & $7.69 \mathrm{E}-4$ & $5.1 \mathrm{E}-5$ \\
0.1 & $3.69 \mathrm{E}-4$ & $3.2 \mathrm{E}-5$ \\
0.3 & $2.42 \mathrm{E}-4$ & $1.9 \mathrm{E}-5$ \\
0.5 & $1.69 \mathrm{E}-4$ & $5.6 \mathrm{E}-5$ \\
\hline
\end{tabular}

\section{List of Figures}

Figure 1 - Dimensions of construction cell and rotor.

Figure 2 - One versus two exponential model for capturing torque/stress decay of fresh mortar (s/c = 2)

Figure 3 - Effect of s/c ratio on parameters $\alpha_{1}$ and $\alpha_{2}$.

Figure 4 - Effect of $\mathrm{s} / \mathrm{c}$ ratio on (a) parameters $\mathrm{M}_{0}, \mathrm{M}_{1}$, and $\mathrm{M}_{2}$ and (b) normalized $\mathrm{M}_{0}, \mathrm{M}_{1}$, and $\mathrm{M}_{2}$.

Figure 5 - Effect of nanoclay addition on parameters $\alpha_{1}$ and $\alpha_{2}$.

Figure 6 - Effect of nanoclay addition on (a) parameters $\mathrm{M}_{0}, \mathrm{M}_{1}$, and $\mathrm{M}_{2}$ and (b) normalized $\mathrm{M}_{0}, \mathrm{M}_{1}$, and $\mathrm{M}_{2}$.

Figure 7 - Effect of angular velocity on parameters $\alpha_{1} \dot{\gamma}$ and $\alpha_{2} \dot{\gamma}$.

Figure 8 - Effect of angular velocity on (a) parameters $\mathrm{M}_{0}, \mathrm{M}_{1}$, and $\mathrm{M}_{2}$ and (b) normalized $\mathrm{M}_{0}, \mathrm{M}_{1}$, and $\mathrm{M}_{2}$. 